\title{
Generation of $\alpha$-Particle Beams With a Multi-kJ, Peta-Watt Class Laser System
}

\begin{abstract}
Daniele Margarone ${ }^{1,2}$, Alessio Morace ${ }^{3}$, Julien Bonvalet ${ }^{4}$, Yuki Abe $^{3}$, Vasiliki Kantarelou ${ }^{2}$, Didier Raffestin ${ }^{4}$, Lorenzo Giuffrida ${ }^{2}$, Philippe Nicolai ${ }^{4}$, Marco Tosca ${ }^{5}$, Antonino Picciotto ${ }^{6}$, Giada Petringa ${ }^{7}$, Giuseppe A. P. Cirrone ${ }^{7}$, Yuji Fukuda ${ }^{8}$, Yasuhiro Kuramitsu ${ }^{9}$, Hideaki Habara ${ }^{9}$, Yasunobu Arikawa $^{3}$, Shinsuke Fujioka ${ }^{3}$, Emmanuel D'Humieres ${ }^{4}$, Georg Korn ${ }^{2}$ and Dimitri Batani ${ }^{4 *}$

${ }^{1}$ Centre for Plasma Physics, Queen's University of Belfast, Belfast, United Kingdom, ${ }^{2}$ ELI-Beamlines, Institute of Physics (FZU), Czech Academy of Sciences, Prague, Czechia, ${ }^{3}$ Institute of Laser Engineering (ILE), Osaka University, Osaka, Japan, ${ }^{4}$ Centre of Intense Lasers and Applications (CELIA), University of Bordeaux, Bordeaux, France, ${ }^{5}$ Physics Department, University of Naples Federico II, Naples, Italy, ${ }^{6}$ Fondazione Bruno Kessler (FBK), Trento, Italy, ${ }^{7}$ Southern National Laboratory (LNS), Istituto Nazionale Fisica Nucleare, Catania, Italy, ${ }^{8}$ Kansai Photon Science Institute, Kyoto, Japan, ${ }^{9}$ Graduate School of Engineering, Osaka University, Osaka, Japan
\end{abstract}

\section{OPEN ACCESS}

Edited by: Eliezer Shalom,

Soreq Nuclear Research Center, Israel

Reviewed by:
Ignazio Licata,
Institute for Scientific Methodology
(ISEM), Italy
Vasileios Basios,
Université libre de Bruxelles, Belgium
*Correspondence:
Dimitri Batani
dimitri.batani@u-bordeaux.fr

Specialty section: This article was submitted to Interdisciplinary Physics, a section of the journal

Frontiers in Physics

Received: 12 May 2020

Accepted: 22 July 2020 Published: 09 September 2020

Citation:

Margarone D, Morace A, Bonvalet J, Abe Y, Kantarelou V, Raffestin D, Giuffrida L, Nicolai P, Tosca M, Picciotto A, Petringa G, Cirrone GAP, Fukuda $Y$, Kuramitsu $Y$, Habara $H$, Arikawa Y, Fujioka S, D'Humieres E, Korn $G$ and Batani D (2020) Generation of $\alpha$-Particle Beams With a Multi-kJ, Peta-Watt Class Laser System. Front. Phys. 8:343. doi: 10.3389/fphy.2020.00343
We present preliminary results on generation of energetic $\alpha$-particles driven by lasers. The experiment was performed at the Institute of Laser Engineering in Osaka using the short-pulse, high-intensity, high-energy, PW-class laser. The laser pulse was focused onto a thin plastic foil (pitcher) to generate a proton beam by the well-known TNSA mechanism which, in turn, was impinging onto a boron-nitride (BN) target (catcher) to generated alpha-particles as a result of proton-boron nuclear fusion events. Our results demonstrate generation of $\alpha$-particles with energies in the range 8-10 $\mathrm{MeV}$ and with a flux around $5 \times 10^{9} \mathrm{sr}^{-1}$.

Keywords: high-intensity lasers, TNSA, CR39, pB fusion, $\alpha$-particles

\section{INTRODUCTION}

In recent years, the interaction of high intensity laser beams with solid targets has paved the way to the generation of bright sources of protons (and different ion species) through the mechanisms of Target Normal Sheath Acceleration (TNSA) [1], including recent achievements with the LFEX PW-laser [2, 3]. Laser driven acceleration of energetic He ions ( $\alpha$-particles) has remained mostly unexplored, with exception of experimental results related to investigation of alternative neutron-less fusion schemes, also known as proton-boron fusion ( $\mathrm{p}-\mathrm{B})$ reaction [4-8]:

$$
p+B^{11} \rightarrow 3 \mathrm{He}^{4}+8.7 \mathrm{MeV}
$$

Although the use of $\mathrm{pB}$ reaction for clean energy production represents an attractive perspective, this would require unpractical temperatures to be sustained through known laser compression geometries, thus beam-target fusion schemes are being proposed. Of course from an energy production point of view, $\mathrm{pB}$ fusion would be the ideal reaction since it does not imply a substantial production of radioactive waste, differently than DT, because there would be no significant neutron emission and hence no activation of the reactor chamber. But fusion reactors based on $\mathrm{pB}$ reaction are clearly not for the near future.

Nevertheless, a strong interest in $\mathrm{pB}$ reactions also lies in the generation of high brilliance $\alpha$-particle sources. Such an interest for laser-driven $\alpha$-particle beams derives from the fact that 
currently there are no available high current $\alpha$-particle sources, considering that they are typically produced from radioactive materials or from accelerating He-ions in large-size cyclotrons. On the other hand, a laser-driven $\alpha$-particle source would be promising due to the high brightness implied by the small size of the laser-plasma interaction point and the short particle bunch duration. If such a source is available, it could be used for important applications, such as:

i medical applications (radioisotope production for cancer therapy or PET);

ii studies related to inertial and magnetic confinement fusion

$\alpha$-sources for radioisotopes production are potentially important because these kinds of sources are rare. The radioisotopes which can be produced by reactions induced by $\alpha$-particles will not only be suitable for medical applications but also for industrial ones (for testing welding for instance).

In fusion, energetic $\alpha$-particles generated in the plasma should sustain self-heating. This means that the penetration length of the $\alpha$-particles must be comparable or smaller than the size of the plasma (in the case of ICF, this is the standard "central hot spot" approach to ignition). It is known that the penetration range of ions in a dense plasma is still not well-established because of the uncertainty in theoretically assessing the Coulomb logarithm which determines the stopping power. In fact, in the last years several experiments have addressed the study of stopping power of protons in plasma and in warm dense matter [9-11]. For $\alpha-$ particles the situation is similar and the effect on the possibility of igniting a thermonuclear target in ICF can be important [12-14]. Thus, development of high-brightness $\alpha$-particle sources is a key step toward quantitatively addressing such open issues through dedicated experiments.

Therefore, although fusion and medical applications are quite far from each other in energy scales and interactions, understanding the mechanisms at the base of laser-driven $\mathrm{pB}$ fusion and developing high-brightness $\alpha$-particle sources will be important for both.

\section{EXPERIMENTAL OBJECTIVES AND METHODS}

In our experiment we studied the possibility of using the highintensity, high-energy, PW-laser LFEX for generating highcurrent, high-energy $\alpha$-particle beams through the p-B nuclear fusion reaction in two different geometries: (i) in-target, and (ii) beam-target nuclear fusion. Both schemes were investigated by using a single-target and a pitcher-catcher setup, respectively. Nevertheless, only results for the beam-target configuration are shown and discussed here.

The scheme of the experimental set-up is sketched in Figure 1, which also schematically shows the different target geometries. In our experiment, the laser was typically delivering $1.4 \mathrm{~kJ}$ in $2.6 \mathrm{ps}$. The calculated focal spot size was $\sim 50 \mu \mathrm{m}$, which corresponds to an intensity on target of $\sim 2-3 \times 10^{19} \mathrm{~W} / \mathrm{cm}^{2}$, taking into account the $\sim 50-60 \%$ encircled energy within the nominal focal spot [3].

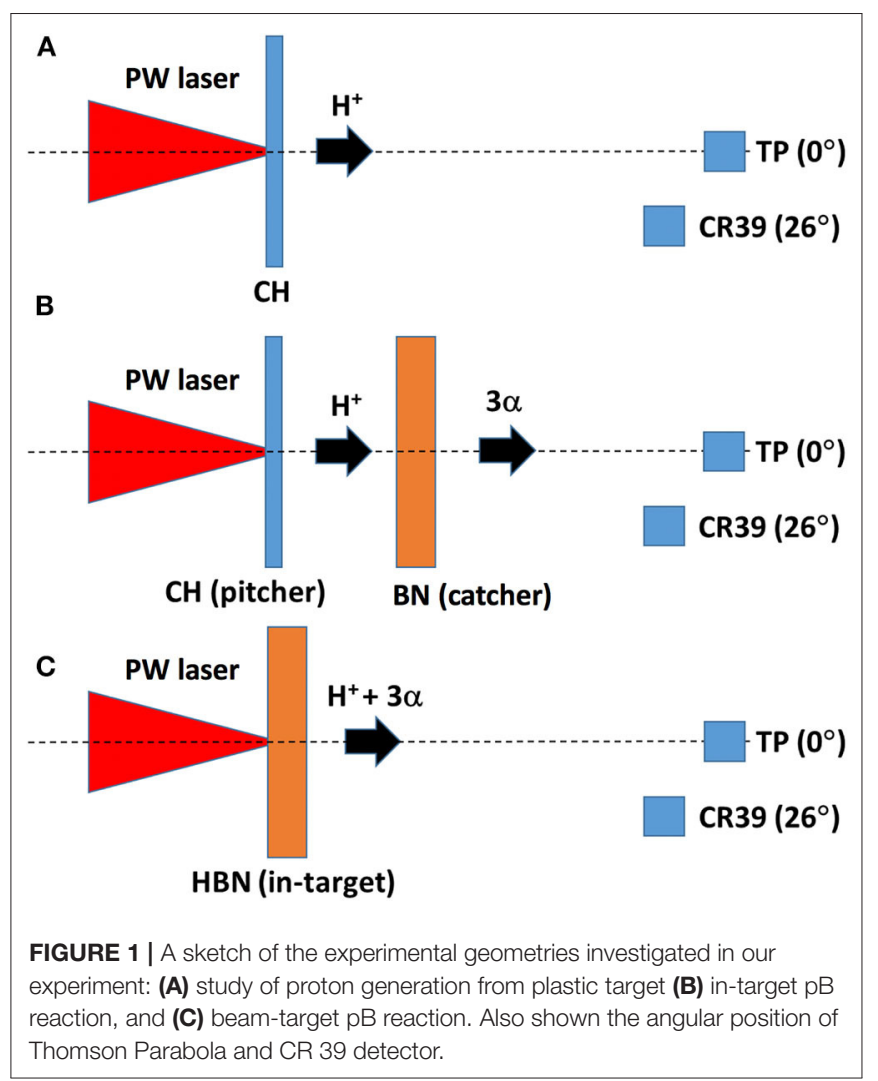

A $25-\mu \mathrm{m}-\mathrm{CH}$ foil was used as pitcher target for the proton acceleration stage (TNSA regime). Then, various catcher targets were tested (2-mm-BN, 0.2-mm-BN, 2-mm-B) to study how the corresponding $\alpha$-particle distribution (particle number vs. energy) is influenced by the target material and thickness (Figure 1B). The in-target $\mathrm{pB}$ reaction mechanism was also investigated by using a $0.2-\mathrm{mm}-\mathrm{BN}$ foil in a single-target geometry (Figure 1A). In this case it is important to notice that Hydrogen is always present in the Boron Nitride target with a concentration of $\sim 1 \%$. This is due to the manufacturing process of $\mathrm{BN}$ which is obtained by reacting trioxide $\left(\mathrm{B}_{2} \mathrm{O}_{3}\right)$ or boric acid $\left(\mathrm{H}_{3} \mathrm{BO}_{3}\right)$ with ammonia $\left(\mathrm{NH}_{3}\right)$ or urea $\left(\mathrm{CO}\left(\mathrm{NH}_{2}\right) 2\right)$ in a nitrogen atmosphere. The content of hydrogen, as a source of protons, is directly related to the chemical synthesis of the material.

Various ion diagnostics were used to determine particle beam energy and number: (i) Thomson Parabola (TP) spectrometer (equipped with an imaging plate) for characterization of accelerated protons; (ii) CR39 nuclear track detectors for detection of generated $\alpha$-particles; (iii) time-of-flight (TOF) diamond detectors for a real-time rough verification of proton and $\alpha$-particle emission; (iv) TOF neutron detector to monitor potential concurrent nuclear reactions.

The TP was facing the target rear side, and was aligned perpendicularly to the target surface and along the laser axis. Instead CR-39 passive detectors were placed at different positions inside the target chamber, facing both the front and rear side of the target, thus allowing to study the angular 


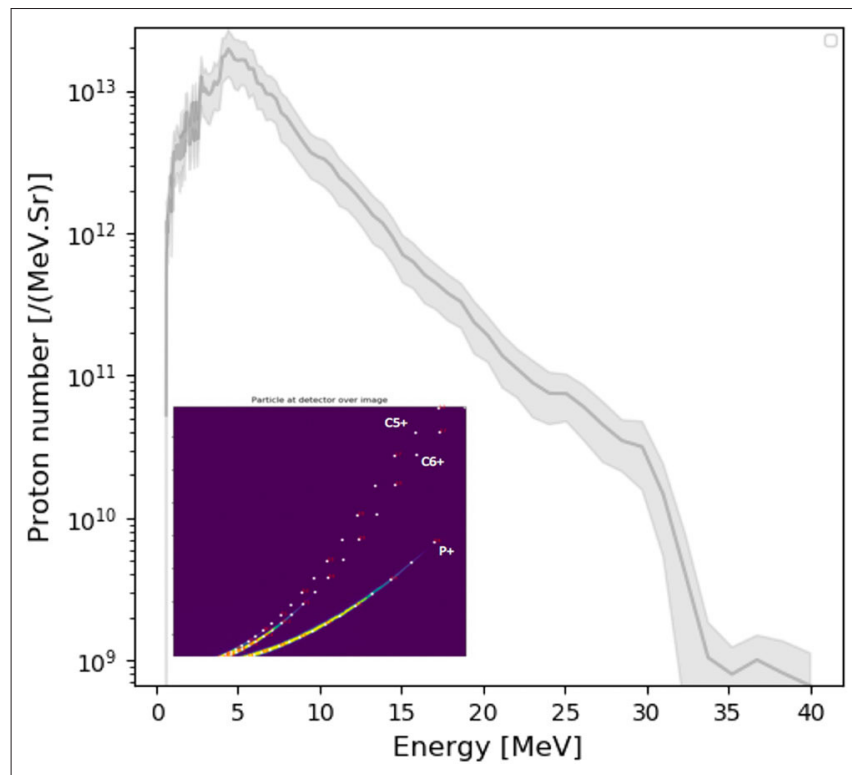

FIGURE 2 | Proton energy distribution measured by the TP spectrometer for the reference shot in which only the $25-\mu \mathrm{m}-\mathrm{CH}$ target was used (laser at 1.4 $\mathrm{kJ} / 2.6 \mathrm{ps})$. This shows acceleration of a very high number of protons $\left(>10^{13}\right.$ $\mathrm{sr}^{-1}$ ) with energies in the 1-10 MeV range. Insets shows the actual ion traces on the IP detector.

distribution of the emitted $\alpha$-particles. The latter was certainly the most reliable diagnostics for a full characterization of the alpha-particle emission, demonstrating outstanding preliminary results, nevertheless their post-mortem analysis is extremely time-consuming. Therefore, in this paper we show preliminary results obtained for the $\mathrm{BN}-2 \mathrm{~mm}$ target and only for the CR39 detector placed on target rear side in proximity of the TP proton spectrometer (at $26^{\circ}$ with respect to the normal to the target, i.e., co-axial to the laser beam). Further experimental results are being analyzed and will be presented in a more comprehensive article currently in preparation.

\section{EXPERIMENTAL RESULTS}

Figure 2 shows the proton energy distribution recorded during the reference shot in which only the $25-\mu \mathrm{m}-\mathrm{CH}$ target was used. This demonstrates acceleration of a very high number of protons $\left(>10^{13} \mathrm{sr}^{-1}\right)$ with energies in the $1-10 \mathrm{MeV}$ range, and still a substantial emission $\left(>10^{11} \mathrm{sr}^{-1}\right)$ in the $10-25 \mathrm{MeV}$ energy range. The estimated laser-to-proton energy conversion efficiency is about $7 \%$.

The energy distribution of protons transmitted through the catcher (configuration with $\mathrm{CH}+2-\mathrm{mm}-\mathrm{BN}$ targets) was also measured and demonstrated that a high number of protons $\left(>10^{11} \mathrm{sr}^{-1}\right)$ accelerated from the pitcher is able to emerge from the catcher rear side with energies in the range of $0.5-2 \mathrm{MeV}$, i.e., in the region where the $\mathrm{pB}$ reaction cross section is maximized (main resonance at $0.7 \mathrm{MeV}$ ).

Figure 3 shows an example of CR39 analysis (optical microscope snapshot) after $30 \mathrm{~min}$. of detector etching. This

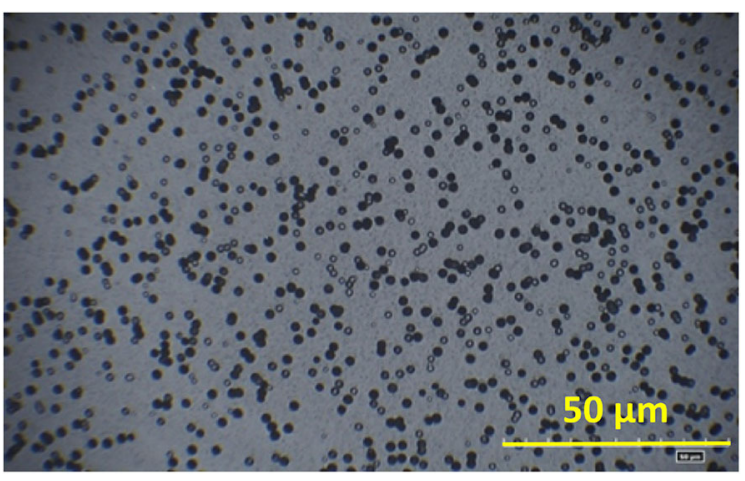

FIGURE 3 | Example of CR39 showing $\alpha$-particle tracks at the optical microscope after 30 min etching. This corresponds to a shot with $\mathrm{CH}+\mathrm{BN}$ pitcher-catcher geometry with a 2-mm thick BN slab. This CR39 sample was facing the catcher rear surface, at an angle of $26^{\circ}$ from the target normal and a detection distance of about $36 \mathrm{~cm}$. The detector was covered with a $30 \mu \mathrm{m} \mathrm{Al}$ foil to stop low energy particles, thus providing a detection threshold energy of 6.4 MeV for $\alpha$-particles. In this shot, the TP spectrum showed the presence of protons with energy $0.5-2 \mathrm{MeV}$.

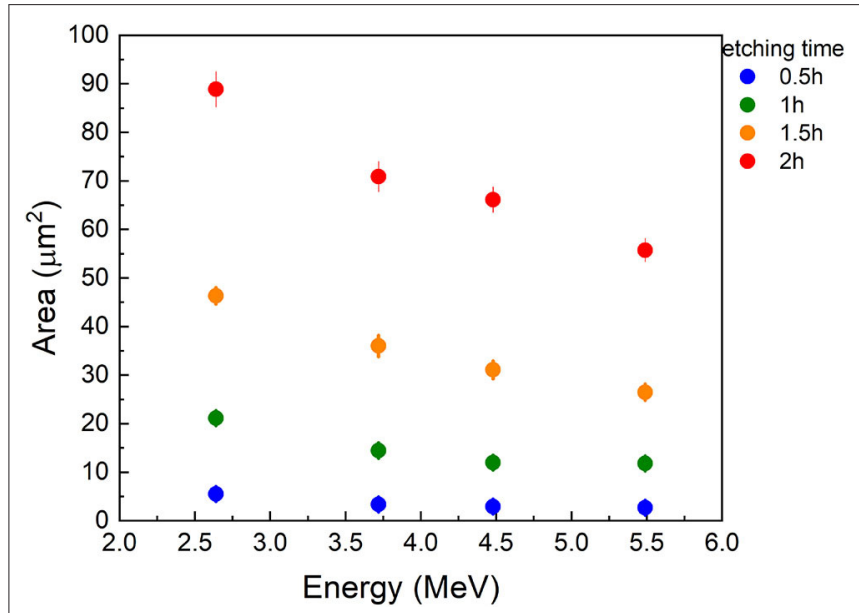

FIGURE 4 | Calibration curve for CR-39 etched using a $\mathrm{KOH} 6 \mathrm{M}$ solution at $70^{\circ} \mathrm{C}$ and using $\alpha$-particles emitted by a $241 \mathrm{Am}$ thin source.

CR39 sample was facing the catcher rear surface, at an angle of $26^{\circ}$ from the target normal and a detection distance of about $36 \mathrm{~cm}$. The detector was covered with a $30 \mu \mathrm{m}$ Al foil to stop low energy particles, thus providing a detection threshold energy of 6.4 MeV for alpha-particles.

CR-39 samples were etched using a $\mathrm{KOH} 6 \mathrm{M}$ solution at $70^{\circ} \mathrm{C}$. In order to obtain the absolute alpha-particle number for a given energy range, we have performed an accurate calibration of the used CR39 detectors using $\alpha$-particles emitted by a $241 \mathrm{Am}$ thin source $(85.2 \%$ with $5.486 \mathrm{MeV}$ ) and $12.8 \%$ with $5.443 \mathrm{MeV}$, and their energy was degraded by using foils of various thicknesses. Our calibration curve correlates $\alpha$-particle track size (in $\mu \mathrm{m}^{2}$ ) with the particle energy for different etching times, as shown in Figure 4. It is worth mentioning that the ion tracks are 
unambiguously caused by $\alpha$-particles because the diameter (i.e., area) of tracks generated by high energy $(>1 \mathrm{MeV})$ protons is much smaller.

Figure 5 shows the alpha particle energy spectrum converted from the flux measured in the CR39 facing the target rear side, averaging over 10 different areas of the same detector. This corresponds to the shot shown in Figure 3. CR39 analysis

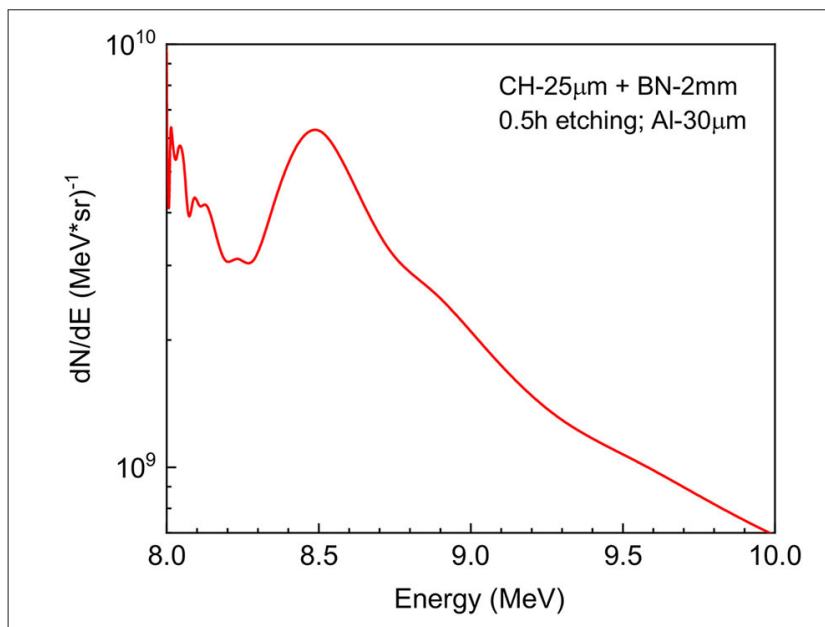

FIGURE 5 | $\alpha$-particle energy spectrum corresponding to Figure $\mathbf{3}$ converted from the flux measured in the CR39 facing the target rear side, averaging over 10 different areas of the same detector. Based on ion track counting technique, the total number of $\alpha$-particles is $5 \times 10^{9} / \mathrm{sr} \pm 20 \%$. unambiguously shows the generation of energetic $\alpha$-particles with energies in the range $8-10 \mathrm{MeV}$. Based on ion track counting technique, the total number of alpha-particles is $5 \times 10^{9} / \mathrm{sr}$ $\pm 20 \%$ in the forward direction (laser propagation axis). This value will have to be compared with results from other target configurations (analysis is in progress).

\section{NUMERICAL SIMULATIONS}

We tried to analyze our experimental results by using advanced simulations. In order to describe the $\alpha$-particle generation in terms of number and energy, we used a chain of numerical simulations. In the first step we performed PIC simulations using the code SMILEI [15] to predict the acceleration of protons in the plastic target (pitcher) mainly by the TNSA mechanism. Such results can be compared with the experimental spectrum shown in Figure 2. In a second step we used the Monte Carlo code FLUKA [16]. In this setup, the protons are sent to the catcher target and $\alpha$-particles are produced through $\mathrm{pB}$ fusion reactions. In this way we separate physical processes of lasermatter interaction and proton generation from those related to $\alpha$-particle production.

Detector signals can be numerically reproduced for each laser shot. We obtained the ion spectra measured by the TP and the $\alpha$-particle fluxes (and energies) measured by the CR-39 as a function of their spatial positions, with the goal to carry out a full comparison and a robust data interpretation. An example of preliminary simulation outputs is shown in Figure 6. For sake of simplicity, this simulation was done with a target of pure B of

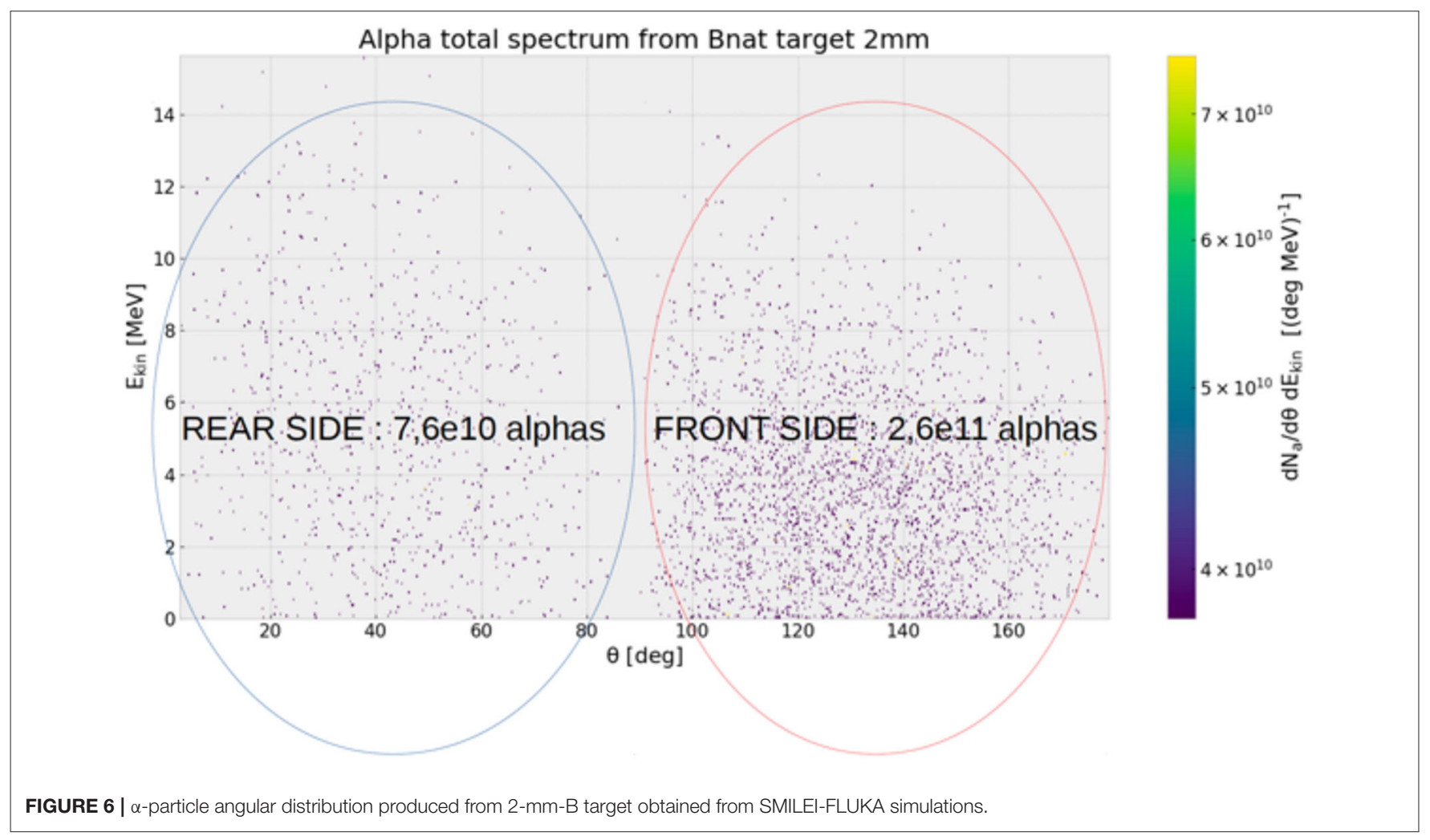


$2 \mathrm{~mm}$ thickness instead of BN $2 \mathrm{~mm}$. The results are qualitatively the same: only, since the target densities are similar, the $\mathrm{BN}$ target contains half boron atoms. As an order of magnitude, we do expect therefore the $\mathrm{BN}$ target to produce half of the $\alpha$-particles of the B target.

\section{CONCLUSIONS}

In our experiment, we studied the generation of energetic $\alpha$-particles driven by using a short-pulse, high-intensity, high-energy, PW-class laser. We used two different target configurations in which $\mathrm{pB}$ fusion reactions were induced either by (i) an external proton beam generated on a secondary $\mathrm{CH}$ target by the TNSA mechanism, or (ii) directly using hydrogen contained in the target which was directly irradiated by the laser. We also tested different thickness of the boron target and different stoichiometries (pure boron vs. boron nitride). Here we have shown only the results obtained for a $2-\mathrm{mm} \mathrm{BN}$ target in the pitcher-catcher configuration. Our results demonstrate generation of $\alpha$-particles with energies in the range $8-10 \mathrm{MeV}$ and with a flux around $5 \times 10^{9} \mathrm{sr}^{-1}$.

\section{DATA AVAILABILITY STATEMENT}

The raw data supporting the conclusions of this article will be made available by the authors, without undue reservation.

\section{REFERENCES}

1. Macchi A, Borghesi M, Passoni M. Ion acceleration by superintense laser-plasma interaction. Rev Mod Phys. (2013) 85:751-93. doi: 10.1103/RevModPhys.85.751

2. Yogo A, Mima K, Iwata N, Tosaki S, Morace A, Arikawa Y, et al. Boosting laser-ion acceleration with multi-picosecond pulses. Sci Rep. (2017) 7:42451. doi: $10.1038 /$ srep 42451

3. Morace A, Iwata N, Sentoku Y, Mima K, Arikawa Y, Yogo A, et al. Enhancing laser beam performance by interfering intense laser beamlets. Nat Comm. (2019) 10:2995. doi: 10.1038/s41467-019-10997-1

4. Belyaev VS, Matafonov AP, Vinogradov VI, Krainov VP, Lisitsa VS, Roussetski AS, et al. Observation of neutronless fusion reactions in picosecond laser plasmas. Phys Rev E. (2005) 72:026406. doi: 10.1103/PhysRevE.72.026406

5. Labaune C, Baccou C, Depierreux S, Goyon C, Loisel G, Yahia V, et al. Fusion reactions initiated by laser accelerated particle beams in a laser-produced plasma. Nat Comm. (2013) 4:2506. doi: 10.1038/ncomms3506

6. Picciotto A, Margarone D, Velyhan A, Bellutti P, Krasa J, Szydlowsky A, et al. Boron-proton nuclear-fusion enhancement induced in boron-doped silicon targets by low-contrast pulsed laser. Phys Rev X. (2014) 4:031030. doi: 10.1103/PhysRevX.4.031030

7. Margarone D, Picciotto A, Velyhan A, Krasa J, Kucharik M, Mangione A, et al. Advanced scheme for high-yield laser driven nuclear reactions. Plasma Phys Control Fusion. (2015) 57:014030. doi: 10.1088/0741-3335/57/1/014030

8. Giuffrida L, Belloni F, Margarone D, Petringa G, Milluzzo G, Scuderi V, et al. High-current stream of energetic $\alpha$ particles from laser-driven proton-boron fusion. Phys Rev E. (2020) 101:013204. doi: 10.1103/PhysRevE.101.013204

9. Chen S N, Atzeni S, Gangolf T, Gauthier M, Higginson DP, Hua R, et al. Experimental evidence for the enhanced and reduced stopping regimes for protons propagating through hot plasmas. Sci Rep. (2018) 8:14586. doi: 10.1038/s41598-018-32726-2

10. Zylstra AB, Frenje JA, Grabowski PE, Li CK, Collins GW, Fitzsimmons P, et al. Measurement of charged-particle stopping in warm dense plasma. Phys Rev Lett. (2015) 114:215002. doi: 10.1103/PhysRevLett.114.215002

\section{AUTHOR CONTRIBUTIONS}

All authors took actively part in the preparation of the experiment, in the analysis of data, and in the writing of the article. In particular DM, AM, DR, PN, GC, GK, and DB prepared the proposal for the experiment. DM, AM, JB, YAb, VK, DR, LG, MT, PN, YF, YK, HH, YAr, and SF took part in the experimental run and took care of diagnostics. DB, LG, and AP took care of target acquisition or preparation. GP and GC performed the calibration of CR39 detectors. JB, PN, and ED'H took care of the numerical simulations.

\section{FUNDING}

This work has been supported by the Ministry of Education, Youth, and Sports of the Czech Republic by the Project No. LQ1606 and by the project Advanced Research Using High Intensity Laser Produced Photons and Particles (CZ.02.1.01/0.0/0.0/16_019/0000789).

\section{ACKNOWLEDGMENTS}

The authors warmly acknowledge the support of the laser team at the Institute of Laser Engineering in running the experiment.

11. Cayzac W, Frank A, Ortner A, Bagnoud V, Basko MM, Bedacht S, et al. Experimental discrimination of ion stopping models near the Bragg peak in highly ionized matter. Nat Comm. (2017) 8:15693. doi: 10.1038/ncomms15693

12. Singleton RL. Charged particle stopping power effects on ignition: some results from an exact calculation. Phys Plasmas. (2008) 15:056302. doi: $10.1063 / 1.2840134$

13. Tahir NA, Piriz AR, Wouchuk G, Shutov A, Lomonosov IV, Fortov VE, et al. Laboratory planetary science studies using intense heavy ion beams at FAIR: the HEDgeHOB collaboration. Nucl Instr Meth Phys Res A. (2009) 606:177-85. doi: 10.1016/j.nima.2009.03.087

14. Temporal M, Canaud B, Cayzac W, Ramis R, Singleton RL Jr. Effects of alpha stopping power modelling on the ignition threshold in a directlydriven inertial confinement fusion capsule. Eur Phys Journ D. (2017) 71:132. doi: 10.1140/epjd/e2017-80126-6

15. Derouillat J, Beck A, Pérez F, Vinci T, Chiaramello M, Grassi A, et al. Smilei: a collaborative, open-source, multi-purpose particle-in-cell code for plasma simulation. Comput Phys Comm. (2018) 222:351-73. doi: 10.1016/j.cpc.2017.09.024

16. Ferrari A, Sala PR, Fasso A, Ranft J. FLUKA: a multi-particle transport code. (2005) 1-387. doi: 10.2172/877507

Conflict of Interest: The authors declare that the research was conducted in the absence of any commercial or financial relationships that could be construed as a potential conflict of interest.

Copyright (C) 2020 Margarone, Morace, Bonvalet, Abe, Kantarelou, Raffestin, Giuffida, Nicolai, Tosca, Picciotto, Petringa, Cirrone, Fukuda, Kuramitsu, Habara, Arikawa, Fujioka, D'Humieres, Korn and Batani. This is an open-access article distributed under the terms of the Creative Commons Attribution License (CC BY). The use, distribution or reproduction in other forums is permitted, provided the original author(s) and the copyright owner(s) are credited and that the original publication in this journal is cited, in accordance with accepted academic practice. No use, distribution or reproduction is permitted which does not comply with these terms. 